\title{
Short-term GDP forecasting with a mixed frequency dynamic factor model with stochastic volatility APPENDIX
}

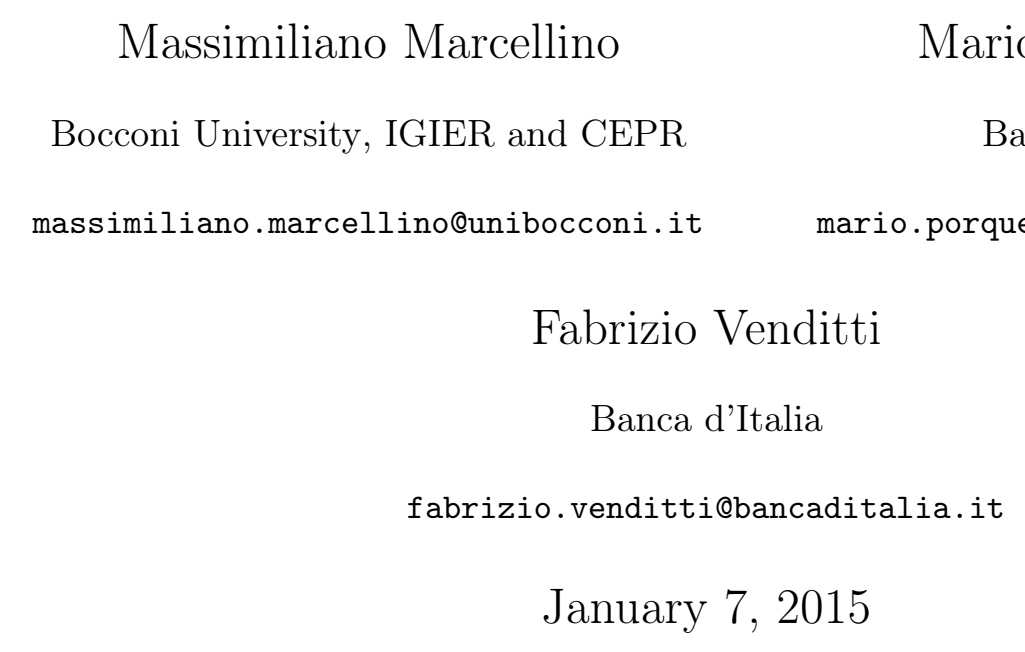

JEL Classification: E32, C22, E27

Keywords: Forecasting, Business cycle, Mixed-frequency data, Nonlinear models, Nowcasting 


\section{A Details of the Gibbs sampler}

We describe in more details the six blocks that compose our Gibbs sampler procedure. The sampler is based on the algorithm by Del Negro and Otrok (2008) modified to account for missing data and mixed frequencies.

\section{A.1 Block 1: drawing the factor loadings $\beta_{q}, \beta_{h}, \beta_{s}$}

In the first block of the Gibbs sampler we draw the factor loadings. Start from the measurement equation of the hard indicator:

$$
y_{h, t}=\beta_{h} f_{t}+u_{h, t}
$$

where the law of motion of the idiosyncratic shock is $u_{h, t}=\phi_{h, 1} u_{h, t-1}+\phi_{h, 2} u_{h, t-2}+\epsilon_{h, t} e^{\lambda_{h, t} / 2}$ and $\epsilon_{h, t} \sim N\left(0, \sigma_{h}\right)$. Since we are conditioning on all the parameters, on the factor $f_{t}$ and on the stochastic volatilities $\lambda_{h, t}$ we treat this as a regression with autocorrelated and heteroscedastic residuals. Now we quasi-difference the equation by filtering both sides with the filter $1-\phi_{h, 1} L-$ $\phi_{h, 1} L^{2}$ and divide each observation by $e^{\lambda_{h, t} / 2}$ :

$$
y_{h, t}^{\star}=\beta_{h} x_{t}^{\star}+\epsilon_{h, t}
$$

where $x_{t}^{\star}=\left(1-\phi_{h, 1} L-\phi_{h, 1} L^{2}\right) f_{t} / e^{\lambda_{h, t} / 2}$. We posit a Normal prior so thath the posterior is also Normal, see Kim and Nelson (1999) for a textbook treatment. The case of survey variables can be treated in the same way after noticing that $x_{t}^{\star}=\left(1-\phi_{h, 1} L-\phi_{h, 1} L^{2}\right) \sum_{j=0}^{11} f_{t-j} / e^{\lambda_{s, t} / 2}$.

In the case of quarterly variables two adjustments are needed. First, since the variable is observed only every three months only these observations can be used for estimating the factor loading. Second, in the measurement equation an MA(4) regression error appears:

$$
y_{q, t}=\beta_{q} w(L) f_{t}+w(L) u_{q, t}
$$

where $w(L)=\frac{1}{3}+\frac{2}{3} L+L^{2}+\frac{2}{3} L^{3}+\frac{1}{3} L^{4}$. Furthermore the error term $u_{t}$ is an $\operatorname{AR}(2)$ process $u_{q, t}=\phi_{q, 1} u_{q, t-1}+\phi_{q, 2} u_{q, t-2}+\epsilon_{q, t} e^{\lambda_{q, t} / 2}$. We work out the variance covariance matrix of the error terms of equation (3), $\Phi\left(\phi_{q, 1}, \phi_{q, 2}, \sigma_{q}^{2}\right)$, which, at this step of the sampler, can be treated as known. Then we divide each observation by $e^{\lambda_{q, t} / 2}$ and pre-multiply both sides of the equation by $\Phi^{-\frac{1}{2}}$ to obtain a standard regression with uncorrelated residuals. Assuming a normal prior, draws of $\beta_{q}$ are obtained from a normal posterior. 


\section{A.2 Block 2: drawing $\phi_{f, 1}, \phi_{f, 2}, \phi_{q, 1}, \phi_{q, 2}, \phi_{h, 1}, \phi_{h, 2}, \phi_{s, 1}, \phi_{s, 2}$}

To draw the AR parameters of the idiosyncratic shocks notice that, conditioning on the state vector $\mu_{t}$, we can treat the common factor $f_{t}$ and the residuals $u_{q, t}, u_{h, t}, u_{s, t}$ as known. The transition equations become standard regression problems which can be analyzed using the same steps used for drawing the factor loadings. We employ normal priors and rule out explosive roots by discarding draws if the roots of $\phi_{j}(L)=0$ lie outside the unit circle.

\section{A.3 Block 3: drawing the innovation variances $\sigma_{f}^{2}, \sigma_{q}^{2}, \sigma_{h}^{2}, \sigma_{s}^{2}$}

We again proceed by treating the transition equations one at the time. Consider a generic element of the state vector $\mu_{i, t}$. Its law of motion is:

$$
\mu_{i, t}=\phi_{i, 1} \mu_{i, t-1}+\phi_{i, 2} \mu_{i, t-2}+\eta_{i, t} \quad \eta_{i, t} \sim N\left(0, \sigma_{i}^{2} e^{\lambda_{i, t}}\right)
$$

For the innovation variance $\sigma_{i}^{2}$ we posit an inverse-Gamma prior $p\left(\sigma_{i}^{2}\right)=I G\left(n_{i}, s_{i}^{2}\right)$. Given our assumption that the idiosyncratic disturbances are normal the posterior is also an inverseGamma, $I G\left(T+n_{i}, \frac{n_{i} s_{i}^{2}+T d_{i}^{2}}{T+n_{i}}\right)$ where:

$$
d_{i}^{2}=\frac{1}{n_{i}} \sum_{t=1}^{n_{i}}\left(\mu_{t, i}-\phi_{i, 1} \mu_{t-1, i}-\phi_{i, 2} \mu_{t-2, i}\right)^{2}
$$

\section{A.4 Block 4: drawing the state vector $\mu_{t}$}

Since the model can be cast in state space draws of the state vector can obtained via a state vector simulation smoother as in Kevin and Kohn (1994) or with the disturbance smoother proposed by Koopman and Durbin (2003). We resort to the latter, which turns out to be slightly more efficient from a computational point of view.

\section{A.5 Block 5: drawing $\lambda_{i, t}$}

To sample the stochastic volatilities $\lambda_{i, t}$ notice that conditional on all parameters and on the states $\mu_{t}$ the orthogonal innovations $\eta_{i, t} / \sigma_{h, i}$ are observable. The $\lambda_{i, t}$ can then be sampled adopting the date-by-date blocking scheme developed by Jacquier et al. (1994). 1 .

\footnotetext{
${ }^{1}$ Details on the algorithm, which involves a Metropolis Hastings step within the Gibbs sampler, can be found in Cogley and Sargent (2005), Appendix B.2.5
} 


\section{A.6 Block 6: drawing $\sigma_{h, i}^{2}$}

The final block of the sampler involves drawing the variances of the log-volatilities. Conditioning on the log-volatilities and postulating an inverse-Gamma prior distribution, the $\sigma_{h, i}^{2}$ can also be drawn from an inverse Gamma posterior.

\section{B The selection of the monthly indicators}

Small scale models have their own "curse of dimensionality": since they rely on a small set of indicators, they are prone to the criticism of potentially leaving out relevant information compared to factor models that use hundreds of time series. Part of the literature has, however, advocated the use of a models of small dimensions. Bai and $\mathrm{Ng}(2008)$ and Boivin and $\mathrm{Ng}$ (2006), for example, question the usefulness of 'too much information' for forecasting purposes, showing that a number of variable selection techniques (already widely used in biomedical statistics where the number of covariates is typically very large) give encouraging results when applied to economic time series. To make the choice of the indicators to be included in our model as objective as possible we proceed as follows. We start by considering a dataset of more than a hundred variables for the period 1987-2011 $2^{2}$, and select a subset of 39 indicators similar to those employed in Angelini et al. (2011) and in Camacho and Perez-Quiros (2010). We then set a priori four core variables that we decide to include in the model, which are Industrial Production for the euro area (IP), the composite Purchasing Manager Index (PMI), the European Commission Economic Sentiment Indicator (ESI) and the Germany IFO Business Climate Index. To select the remaining variables, we calculate as a benchmark the percentage of GDP variance explained by the factor computed from the core variables only, as in Camacho and Perez-Quiros (2010), and design an algorithm for the selection of a set of additional indicators which maximize this statistic.

1. We evaluate datasets with all core variables and one other variable at a time in order to calculate the explained variance, and the probability that it is higher than in the dataset with core variables only. In this way we obtain a ranking of the other series.

2. We add a variable at a time, starting with the ones with an higher probability to increase the explained variance with respect to the benchmark; we keep the variable only if this probability increases. We end up with the small set of 8 variables described in the main text.

\footnotetext{
${ }^{2}$ The series are those used to compile $€$-Coin (see Altissimo et al. (2010). For a description of the dataset see http://eurocoin.bancaditalia.it/
} 


\section{The state space specification in the empirical application}

The specification we adopt follows Camacho and Perez-Quiros (2010) where surveys are modeled as a 12 terms moving average of the unobserved factor, while hard variables load the factor contemporaneously. This amounts to imposing that surveys are in phase with the year on year growth rate of Industrial Production (and of the other hard indicators). To get an idea of the state representation of the model while keeping notation to a minimum we present the case of a toy model with one quarterly variable, one hard indicator and one soft indicator in which all the idiosyncratic shocks follow an $\mathrm{AR}(2)$ process. The more general case can be easily derived from this example. The loading matrix $\mathrm{F}$ in the measurement equation (??) can be written as:

$F=\left(\begin{array}{ccccccccccccccccccccc}\beta_{q} \frac{1}{3} & \beta_{q} \frac{2}{3} & \beta_{q} & \beta_{q} \frac{2}{3} & \beta_{q} \frac{1}{3} & 0 & 0 & 0 & 0 & 0 & 0 & 0 & \frac{1}{3} & \frac{2}{3} & 1 & \frac{2}{3} & \frac{1}{3} & 0 & 0 & 0 & 0 \\ \beta_{h} & 0 & 0 & 0 & 0 & 0 & 0 & 0 & 0 & 0 & 0 & 0 & 0 & 0 & 0 & 0 & 0 & 1 & 0 & 0 & 0 \\ \beta_{s} & \beta_{s} & \beta_{s} & \beta_{s} & \beta_{s} & \beta_{s} & \beta_{s} & \beta_{s} & \beta_{s} & \beta_{s} & \beta_{s} & \beta_{s} & 0 & 0 & 0 & 0 & 0 & 0 & 0 & 1 & 0 \\ & & & & & & & & & & & & & & & & & & & & \end{array}\right)$

where $\beta_{q}, \beta_{h}$ and $\beta_{s}$ are the loadings of, respectively, the quarterly variable, the hard and the soft indicators. The state vector is:

$$
\mu_{t}=\left(\begin{array}{lllllllllll}
f_{t} & f_{t-1} & \ldots & f_{t-11} & u_{q, t} & \ldots & u_{q, t-4} & u_{h, t} & u_{h, t-1} & u_{s, t} & u_{s, t-1}
\end{array}\right)^{\prime}
$$

The transition matrix is:

$$
H=\left(\begin{array}{rrrrrrrrrrrrrrr}
\phi_{f, 1} & \phi_{f, 2} & 0 & 0 & 0 & \ldots & 0 & 0 & 0 & 0 & 0 & 0 & 0 & 0 & 0 \\
1 & 0 & 0 & 0 & 0 & \ldots & 0 & 0 & 0 & 0 & 0 & 0 & 0 & 0 & 0 \\
0 & 0 & 0 & 0 & 0 & \ldots & 0 & 0 & 0 & 0 & 0 & 0 & 0 & 0 & 0 \\
\vdots & \vdots & \vdots & \vdots & \vdots & \vdots & \vdots & \vdots & \vdots & \vdots & \vdots & \vdots & \vdots & \vdots & \vdots \\
0 & 0 & 0 & 0 & 0 & \ldots & \phi_{q, 1} & \phi_{q, 2} & 0 & 0 & 0 & 0 & 0 & 0 & 0 \\
0 & 0 & 0 & 0 & 0 & \ldots & 1 & 0 & 0 & 0 & 0 & 0 & 0 & 0 & 0 \\
0 & 0 & 0 & 0 & 0 & \ldots & 0 & 0 & 0 & 0 & 0 & 0 & 0 & 0 & 0 \\
\vdots & \vdots & \vdots & \vdots & \vdots & \vdots & \vdots & \vdots & \vdots & \vdots & \vdots & \vdots & \vdots & \vdots & \vdots \\
0 & 0 & 0 & 0 & 0 & \ldots & 0 & 0 & 0 & 0 & 0 & \phi_{h, 1} & \phi_{h, 2} & 0 & 0 \\
0 & 0 & 0 & 0 & 0 & \ldots & 0 & 0 & 0 & 0 & 0 & 1 & 0 & 0 & 0 \\
0 & 0 & 0 & 0 & 0 & \ldots & 0 & 0 & 0 & 0 & 0 & 0 & 0 & \phi_{s, 1} & \phi_{s, 2} \\
0 & 0 & 0 & 0 & 0 & \ldots & 0 & 0 & 0 & 0 & 0 & 0 & 0 & 1 & 0 \\
& & & & & & & & & & & & & &
\end{array}\right)
$$


Since the idiosyncratic shocks are collected in the state vector the matrix $R_{t}$ is a $(\mathrm{k}+2)$ dimension zero matrix while the matrix $Q_{t}$ is a diagonal matrix which collects all the variances:

$$
Q_{t}=\operatorname{diag}\left(\begin{array}{ccccccccccccccc}
1 & 0 & 0 & 0 & \ldots & \sigma_{q}^{2} e^{\lambda_{q, t}} & 0 & 0 & 0 & 0 & \sigma_{h}^{2} e^{\lambda_{h, t}} & 0 & \sigma_{s}^{2} e^{\lambda_{s, t}} & 0 \\
& & & & & & & & & & & & &
\end{array}\right)
$$

\section{C.1 The model with two factors}

As a robustness check we have extend the baseline model to include a second factor, which we model as a (restricted) $\operatorname{ARMA}(2,2)$ process as in Frale et al. (2011). The two monthly unobserved factors have the following reduced form representations:

$$
\begin{aligned}
& \left(1-\varphi_{11} L-\varphi_{12} L^{2}\right) f_{1, t}=\varepsilon_{1 t} \\
& \left(1-\varphi_{21} L-\varphi_{22} L^{2}\right) f_{2, t}=(1-\theta L)^{2} \varepsilon_{2 t}
\end{aligned}
$$

where $\varepsilon_{1 t} \sim N\left(0, \sigma_{1 \varepsilon}\right)$ and $\varepsilon_{2 t} \sim N\left(0, \sigma_{2 \varepsilon}\right)$. Frale et al. (2009) set $\theta=0.5$, motivating such restriction as a way to enhance the fit at low frequencies, see also Morton and Tunncliffe-Wilson (2004). We sketch the State Space representation of the modified model in a simple setup with three indicators (GDP, a hard monthly variable and a soft monthly variable). Since the presence of these extra MA terms produces a smoother factor we drop the 12 terms moving average representation of the loadings of the surveys in favour of a more standard contemporaneous relationship, so that the measurement matrix $F$ is composed of the three following blocks:

$$
\begin{gathered}
F_{1}=\left(\begin{array}{cccccccc}
\beta_{q, 1} \frac{1}{3} & \beta_{q, 1} \frac{2}{3} & \beta_{q, 1} & \beta_{q, 1} \frac{2}{3} & \beta_{q, 1} \frac{1}{3} \\
\beta_{h, 1} & 0 & 0 & 0 & 0 \\
\beta_{s, 1} & 0 & 0 & 0 & 0
\end{array}\right) \\
F_{2}=\left(\begin{array}{cccccccc}
\beta_{q, 2} \frac{1}{3} & \beta_{q, 2} \frac{2}{3} & \beta_{q, 2} & \beta_{q, 2} \frac{2}{3} & \beta_{q, 2} \frac{1}{3} & 0 & 0 \\
\beta_{h, 2} & 0 & 0 & 0 & 0 & 0 & 0 \\
\beta_{s, 2} & 0 & 0 & 0 & 0 & 0 & 0
\end{array}\right) \\
F_{3}=\left(\begin{array}{ccccccccc}
\frac{1}{3} & \frac{2}{3} & 1 & \frac{2}{3} & \frac{1}{3} & 0 & 0 & 0 & 0 \\
0 & 0 & 0 & 0 & 0 & 1 & 0 & 0 & 0 \\
0 & 0 & 0 & 0 & 0 & 0 & 0 & 1 & 0
\end{array}\right)
\end{gathered}
$$

where $F_{1}$, and $F_{2}$ collect the loadings on the first and second factor and $F_{3}$ the loadings on the idiosyncratic disturbances, which are also modelled as $A R(2)$ processes. Notice that $F_{2}$ has two extra column vectors of zeros, necessary to accommodate the MA terms in the second 
factor.

$$
F=\left[F_{1}, F_{2}, F_{3}\right]
$$

We adopt the $\max (p, q+1)$ representation, see Durbin and Koopman (2006), which requires the slightly more general specification of the transition equations:

$$
\mu_{t}=T \mu_{t-1}+R \eta_{t}
$$

where $\eta_{t} \sim\left(0, Q_{t}\right), Q_{t}$ is a 5 dimensional diagonal matrix (collecting the variances of the 5 idiosyncratic terms, 3 for the observable indicators, 2 for the unobserved factors) and $R$ is a $21 \times 5$ selection matrix. The matrix $T$ is block diagonal with the following blocks:

$$
\begin{aligned}
T_{1}= & {\left[\begin{array}{ccccc}
\varphi_{11} & \varphi_{12} & 0 & 0 & 0 \\
1 & 0 & 0 & 0 & 0 \\
0 & 1 & 0 & 0 & 0 \\
0 & 0 & 1 & 0 & 0 \\
0 & 0 & 0 & 1 & 0
\end{array}\right], T_{2}=\left[\begin{array}{ccccccc}
\varphi_{21} & 0 & 0 & 0 & 0 & 1 & 0 \\
1 & 0 & 0 & 0 & 0 & 0 & 0 \\
0 & 1 & 0 & 0 & 0 & 0 & 0 \\
0 & 0 & 1 & 0 & 0 & 0 & 0 \\
0 & 0 & 0 & 1 & 0 & 0 & 0 \\
\varphi_{22} & 0 & 0 & 0 & 0 & 0 & 1 \\
0 & 0 & 0 & 0 & 0 & 0 & 0
\end{array}\right], } \\
T_{3}= & {\left[\begin{array}{ccccc}
\varphi_{q, 1} & \varphi_{q, 2} & 0 & 0 & 0 \\
1 & 0 & 0 & 0 & 0 \\
0 & 1 & 0 & 0 & 0 \\
0 & 0 & 1 & 0 & 0 \\
0 & 0 & 0 & 1 & 0
\end{array}\right], T_{4}=\left[\begin{array}{cccccc}
\varphi_{h, 1} \\
1 & \varphi_{h, 2} & & \\
1 & 0 &
\end{array}\right], T_{5}=\left[\begin{array}{c}
\varphi_{s, 1} \\
1
\end{array}\right] }
\end{aligned}
$$

the state vector is 21 dimensional:

$$
\mu_{t}=\left[f_{1 t}, f_{1 t-1}, \ldots, f_{1 t-4}, f_{2 t}, f_{2 t-1}, \ldots f_{2 t-4}, z_{1, t}, z_{2, t}, u_{q t}, u_{q t-1}, \ldots, u_{q t-4}, u_{h t}, u_{h t-1}, u_{s t}, u_{s t-1}\right]
$$


and the selection matrix $R$ is

$$
R=\left[\begin{array}{ccccc}
1 & 0 & 0 & 0 & 0 \\
0 & 0 & 0 & 0 & 0 \\
0 & 0 & 0 & 0 & 0 \\
0 & 0 & 0 & 0 & 0 \\
0 & 0 & 0 & 0 & 0 \\
0 & 1 & 0 & 0 & 0 \\
0 & 0 & 0 & 0 & 0 \\
0 & 0 & 0 & 0 & 0 \\
0 & 0 & 0 & 0 & 0 \\
0 & 0 & 0 & 0 & 0 \\
0 & -2 \theta & 0 & 0 & 0 \\
0 & \theta^{2} & 0 & 0 & 0 \\
0 & 0 & 1 & 0 & 0 \\
0 & 0 & 0 & 0 & 0 \\
0 & 0 & 0 & 0 & 0 \\
0 & 0 & 0 & 0 & 0 \\
0 & 0 & 0 & 0 & 0 \\
0 & 0 & 0 & 1 & 0 \\
0 & 0 & 0 & 0 & 0 \\
0 & 0 & 0 & 0 & 1 \\
0 & 0 & 0 & 0 & 0
\end{array}\right]
$$

It can be seen that the two additional state variables $z_{t}^{1}$ and $z_{t}^{2}$ are:

$$
\begin{aligned}
z_{1, t} & =\varphi_{22} f_{2, t-1}+z_{2, t-1}-2 \theta \varepsilon_{t} \\
z_{2, t} & =\theta^{2} \varepsilon_{t}
\end{aligned}
$$

Finally, $Q_{t}=\left(1, \sigma_{f_{2}}^{2} e^{\lambda_{f_{2}, t}}, \sigma_{q}^{2} e^{\lambda_{q, t}}, \sigma_{h}^{2} e^{\lambda_{h, t}}, \sigma_{s}^{2} e^{\lambda_{s, t}}\right)$.

In our empirical application, however, this second factor is not well identified. In fact, as shown in Table 1, the loadings of the indicators on the second factor collapse to zero, with the first factor accounting for the co-movement in the data.

\section{News and forecast revisions}

In their paper Banbura and Modugno (2014) derive a way to decompose a forecast revision as 
a linear function of news.

They denote as $\Omega_{v}$ a vintage of data corresponding to a statistical data release $\mathrm{v}$, which as an example can be mid-month for industrial production and end of month for surveys, in order to define news as:

$$
I_{v+1, j}=y_{i_{j}, t_{j}}-E\left[y_{i_{j}, t_{j}} \mid \Omega_{v}\right]
$$

the surprise incorporated in a new data with respect to what was expected given information $\Omega_{v}$. A forecast revision is defined as:

$$
E\left[y_{k, t_{k}} \mid I_{v+1}\right]=E\left[y_{k, t_{k}} \mid \Omega_{v+1}\right]-E\left[y_{k, t_{k}} \mid \Omega_{v}\right]
$$

and can be expressed as weighted average of news:

$$
E\left[y_{k, t_{k}} \mid I_{v+1}\right]=B_{v+1} I_{v+1}=E\left[y_{k, t_{k}} I_{v+1}^{\prime}\right] E\left[I_{v+1} I_{v+1}^{\prime}\right]^{-1} I_{v+1}
$$

where:

$$
\begin{aligned}
& E\left[y_{k, t_{k}} I_{v+1, j}\right]=H_{k} E\left[\left(\mu_{t_{k}}-E\left(\mu_{t_{k}} \mid \Omega_{v}\right)\right)\left(\mu_{t_{j}}-E\left(\mu_{t_{j}} \mid \Omega_{v}\right)^{\prime}\right)\right] H_{i_{j}}^{\prime} \\
& E\left[I_{v+1, j} I_{v+1, l}\right]=H_{i_{j}} E\left[\left(\mu_{t_{j}}-E\left(\mu_{t_{j}} \mid \Omega_{v}\right)\right)\left(\mu_{t_{l}}-E\left(\mu_{t_{l}} \mid \Omega_{v}\right)^{\prime}\right)\right] H_{i_{l}}^{\prime}
\end{aligned}
$$

where $E\left[\left(\mu_{t_{j}}-E\left(\mu_{t_{j}} \mid \Omega_{v}\right)\right)\left(\mu_{t_{l}}-E\left(\mu_{t_{l}} \mid \Omega_{v}\right)^{\prime}\right)\right]$ is the state vector covariance matrix obtained as a by-product of the Kalman Smoother. 
Table 1: Factor loadings, two factor model

\begin{tabular}{lcccccc}
\hline \multicolumn{1}{c}{ Percentiles } & \multicolumn{3}{c}{ First factor } & \multicolumn{3}{c}{ Second factor } \\
\hline & 75 th & 50 th & 25 th & 75 th & 50 th & 25 th \\
GDP & 0.44 & 0.27 & 0.08 & 0.00 & 0.00 & 0.00 \\
Industrial Production & 0.67 & 0.55 & 0.41 & 0.00 & 0.00 & 0.00 \\
Industrial Production - Pulp/paper & 0.34 & 0.28 & 0.21 & 0.00 & 0.00 & 0.00 \\
Business Climate - IFO & 0.04 & 0.02 & 0.00 & 0.00 & 0.00 & 0.00 \\
Economic Sentiment Indicator & 0.03 & 0.01 & -0.01 & 0.00 & 0.00 & 0.00 \\
PMI composite & 0.02 & 0.01 & -0.01 & 0.00 & 0.00 & 0.00 \\
dollar-euro & 0.01 & -0.01 & -0.04 & 0.00 & 0.00 & 0.00 \\
10y-3m spread & 0.03 & 0.01 & 0.00 & 0.00 & 0.00 & 0.00 \\
Michigan Consumer Sentiment & 0.09 & 0.05 & 0.02 & 0.00 & 0.00 & 0.00 \\
\hline
\end{tabular}




\section{References}

Altissimo, Filippo, Riccardo Cristadoro, Mario Forni, Marco Lippi, and Giovanni Veronese (2010), "New eurocoin: Tracking economic growth in real time." The Review of Economic and Statistics, 92, 1024-1034.

Angelini, Elena, Gonzalo Camba-Mendez, Domenico Giannone, Lucrezia Reichlin, and Gerhard Ruenstler (2011), "Short-term forecasts of euro area gdp growth." Econometrics Journal, 14, $\mathrm{C} 25-\mathrm{C} 44$.

Bai, Jushan and Serena Ng (2008), "Forecasting economic time series using targeted predictors." Journal of Econometrics, 146, 304-317.

Banbura, Marta and Michele Modugno (2014), "Maximum likelihood estimation of factor models on datasets with arbitrary pattern of missing data." Journal of Applied Econometrics, 29, 133-160.

Boivin, Jean and Serena Ng (2006), "Are more data always better for factor analysis?" Journal of Econometrics, 132, 169-194.

Camacho, Maximo and Gabriel Perez-Quiros (2010), "Introducing the euro-sting: Short-term indicator of euro area growth." Journal of Applied Econometrics, 25, 663-694.

Cogley, Timothy and Thomas J. Sargent (2005), "Drift and volatilities: Monetary policies and outcomes in the post wwii u.s." Review of Economic Dynamics, 8, 262-302.

Del Negro, Marco and Christopher Otrok (2008), "Dynamic factor models with time-varying parameters: measuring changes in international business cycles." Staff Reports 326, Federal Reserve Bank of New York.

Frale, Cecilia, Massimiliano Marcellino, Gian Luigi Mazzi, and Tommaso Proietti (2011), "Euromind: a monthly indicator of the euro area economic conditions." Journal of the Royal Statistical Society Series A, 174, 439-470.

Jacquier, Eric, Nicholas G. Polson, and Peter E. Rossi (1994), "Bayesian analysis of stochastic volatility models." Journal of Business \&6 Economic Statistics, 12, 371-89.

Kevin, Carter and Robert Kohn (1994), "On gibbs sampling for state space models." Biometrika, 81, 541-553.

Kim, C. and R. Nelson (1999), State Space Models with Regime Switching. MIT Press, Cambridge, Massachussets. 
Koopman, S. J. and J. Durbin (2003), "Filtering and smoothing of state vector for diffuse state-space models." Journal of Time Series Analysis, 24, 85-98. 